\title{
Vas Deferens Obstruction
}

National Cancer Institute

\section{Source}

National Cancer Institute. Vas Deferens Obstruction. NCI Thesaurus. Code C78706.

Blockage of the normal flow of the sperm in the vas deferens. 